\title{
Alimentación sostenida durante diarrea aguda en niños menores de cinco años
}

\author{
Homero Martínez-Salgado, M.C., Ph.D., (1) \\ Silvia Diez-Urdanivia, Lic. en N utr., (2) Luz María Meneses-Díaz, T.S.(2)
}

\begin{abstract}
Martínez-Salgado $\mathrm{H}$,
Diez-Urdanivia S, Meneses-Díaz LM. Alimentación sostenida durante diarrea aguda en niños menores de cinco años. Salud Publica Mex 1998;40:141-149.
\end{abstract}

\section{Resumen}

Objetivo. Identificar alimentos locales con aceptabilidad cultural para nutrir a niños con diarrea aguda y probar su aceptación e impacto sobre el peso corporal en niños enfermos. Material y métodos. Se entrevistó a 142 madres de niños menores de cinco años en comunidades rurales, para conformar dietas culturalmente aceptables mediante sorteo de grupos, desplegado en un diagrama multidimensional. Se hizo un ensayo sobre el curso clínico de 54 niños de 4-50 meses de edad cursando las primeras 48 horas de un episodio diarreico, quienes recibieron las dietas desarrolladas. Resultados. Se diseñaron dietas de acuerdo con la edad del niño y con el tiempo de evolución de la enfermedad. En las $47.6 \pm 22.2$ horas que permanecieron hospitalizados, los niños consumieron $44.8 \pm 28.6 \mathrm{kcal} / \mathrm{kg} / \mathrm{dí}$ (además de la leche materna) y ganaron $70.6 \pm 179.7 \mathrm{~g}$. Hubo una relación directa entre mayor edad y mayor consumo calórico, y entre éste y mayor ganancia de peso. Conclusiones Los niños mostraron buena aceptabilidad a las dietas ofrecidas, de tal manera que se evitó la pérdida de peso.

Palabras clave: alimentación sostenida; diarrea infantil; niño; México

\section{Martínez-Salgado $\mathrm{H}$,}

Diez-Urdanivia S, Meneses-Díaz LM.

Sustained feeding during acute diarrhea in children younger than five years of age.

Salud Publica Mex 1998;40:141-149.

\begin{abstract}
A bstract
Objective. To identify locally available and culturally acceptable foods for children with acute diarrhea, and test their acceptance and effect on the child's weight. Material and methods. 142 mothers of children younger than five years of age living in rural communities were interviewed to indentify culturally accepted diets by means of group sorting. These were displayed in a multidimensional scale. A descriptive study was performed of the clinical course of 54 children, ages 4 to 50 months who received the designed diets during the first 48 hours of diarrhea. Results. The designed diets considered age of the child, and stage of the disease. Children spent $47.6 \pm 22.2 \mathrm{~h}$ in the hospital during which they consumed $44.8 \pm 28.6 \mathrm{kcal} / \mathrm{kg} /$ day (additional to breast milk) and they gained $70.6 \pm 179.7 \mathrm{~g}$. A direct relationship was observed between increasing age and larger caloric intake, and between this and greater weight gain. Conclusion. Children showed good acceptance of diets. Caloric intake was enough to prevent weight loss.
\end{abstract}

Key words: sustained feeding; infantile diarrhea; child; Mexico

(1) Grupo Interinstitucional de Investigación en Sistemas de Salud SSA-IMSS; C entro de Investigación en Salud Poblacional, Instituto N acional de Salud Pública, México.

(2) Subdirección General de Nutrición, Instituto N acional de la Nutrición Salvador Zubirán, México.

Fecha de recibido: 10 de julio de 1997 • Fecha de aprobado: 28 de enero de 1998

Solicitud de sobretiros: Dr. Homero Martínez Salgado. Cardenales 76, colonia Las A guilas, 01710 México, D.F. Correo electrónico: homero@ profmexis.sar.net 
$\mathrm{L}$ a diarrea es un problema de salud pública en los países en desarrollo, por su alta carga de morbilidad y mortalidad. ${ }^{1}$ En México, la diarrea aguda es la primera causa de mortalidad en niños de entre 1 y 5 años, ${ }^{2,3}$ y se relaciona con las complicaciones de la enfermedad, entre las cuales sobresale la pérdida aguda de agua y electrolitos. ${ }^{4,5}$ Entre los sobrevivientes, los episodios de diarrea pueden causar, precipitar o exacerbar la desnutrición, tanto de macro como de micronutrientes. ${ }^{6}$ Los efectos deletéreos de la diarrea sobre el estado nutricio del niño se producen por uno o más de los siguientes mecanismos: reducción en la ingestión dietética, ${ }^{7-10}$ absorción disminuida de nutrientes, ${ }^{11-13}$ aumento del catabolismo, ${ }^{14}$ pérdida directa de nutrientes por el intestino ${ }^{8,15,16}$ e ineficiencia metabólica debida a deficiencias de micronutrientes. ${ }^{17-19}$

En diversos estudios se ha demostrado que, entre las ventajas de la alimentación sostenida durante la diarrea, están la recuperación más rápida de la fisiología alterada, incluyendo la absorción intestinal;,20, 21 asimismo, los episodios diarreicos se hacen más cor$\operatorname{tos}^{22-24} \mathrm{y}$ hay mayor ganancia de peso de los niños durante y después del episodio diarreico. ${ }^{25}$ Con base en estas evidencias, la recomendación actual de la Organización Mundial de la Salud es que se mantenga la alimentación durante el episodio diarreico, dando preferencia a los alimentos a los que el niño esté habituado. ${ }^{26}$ La mayoría de estos estudios están enfocados ya sea al uso de fórmulas lácteas hidrolizadas o elementales, o bien, los alimentos específicos, pero en ningún caso en el contexto de una dieta que se propone a las madres para que la preparen en el hogar. En México es insuficiente la información respecto al uso de dietas basadas en alimentos disponibles localmente y que las madres acepten para alimentar a sus hijos durante la enfermedad, especialmente en el ámbito comunitario. Esta información es importante para poder adecuar las recomendaciones que debe hacer el personal de salud a las madres respecto a la alimentación de sus hijos durante la enfermedad.

El presente estudio se centró en estos puntos, siguiendo una secuencia en dos fases. El objetivo de la primera fase (1988) fue identificar las creencias de las madres acerca de la alimentación infantil durante episodios de la diarrea aguda, particularizando respecto a alimentos específicos que fueran ofrecidos y suspendidos. Como resultado de esta fase se eligieron alimentos disponibles en la localidad a fin de conformar una dieta culturalmente aceptable y que los niños con diarrea consumieran. La segunda fase (1989) consistió en probar la aceptabilidad de los niños a dicha dieta, así como el efecto de la alimentación sostenida sobre el peso de los niños durante un episodio de diarrea aguda. Esta fase se desarrolló en una clínica ubicada en la misma área de estudio.

\section{Material y métodos}

El área de estudio abarcó ocho comunidades rurales del Estado de México, en la región conocida como Valle de Solís, donde confluyen el Estado de México, Michoacán y Querétaro. Las comunidades son de origen mestizo y hablan español; su economía y su alimentación dependen de la agricultura, orientada fundamentalmente a la siembra de maíz para el consumo familiar. $^{27}$

\section{Estudio etnográfico}

La primera fase consistió en un estudio etnográfico basado en entrevistas con las madres en el hogar. El desarrollo del cuestionario aplicado se basó en un estudio piloto, en el que se entrevistó a informantes clave, para seleccionar los términos adecuados y ayudar a estructurar las preguntas. ${ }^{28}$ Una vez diseñado el cuestionario, se aplicó a la población de estudio, misma que se seleccionó a partir de un censo que se hizo en las comunidades, y en el cual se identificó a todas las madres con al menos un niño menor de cinco años. De éstas, se seleccionó aleatoriamente a 160 madres para entrevistarlas, estratificando el muestreo con base en la edad de las mujeres (15-24 años, 25-34 años, > 34 años) y alfabetismo o analfabetismo.

Una vez que se obtuvo el listado de alimentos disponibles en la comunidad y que las madres estaban dispuestas a ofrecer a sus hijos durante los episodios de diarrea aguda, se procedió a diseñar diversas dietas basadas en dichos alimentos, buscando conformar menús que tuvieran sentido cultural para ellas. Se llevó a cabo un ejercicio de sorteo libre de alimentos con una submuestra de $25 \%$ de las madres entrevistadas, elegida en forma aleatoria, para buscar las asociaciones entre los alimentos que ellas aceptaban darles a sus hijos cuando tenían diarrea. Así se elaboraron una serie de dibujos de los alimentos que al menos dos terceras partes de las madres entrevistadas habían mencionado como aceptables para sus hijos, y se le presentaron a cada una, pidiéndole que formara grupos con los alimentos teniendo en mente la alimentación para un niño con diarrea. La técnica del sorteo libre consiste en mostrar a los informantes una serie de tarjetas con los nombres o los dibujos (como en este caso) de los elementos que se van a sortear, y en pedirles que los acomoden por grupos. Se permite cualquier cantidad de grupos, 
a excepción de formar uno solo o de que cada elemento esté en un grupo por sí mismo. ${ }^{29}$ Los alimentos para el sorteo se eligieron entre aquellos aceptados al menos por dos terceras partes de las madres. Una entrevistadora, previamente adiestrada en la técnica, pidió a las madres que sortearan los alimentos de acuerdo con su propia interpretación, bajo el criterio de "qué alimentos van juntos".

\section{Estudio clínico}

A partir de los resultados del análisis multidimensional se diseñaron dietas adaptadas a la edad del niño y al tiempo de evolución de la enfermedad. En esta fase del estudio se ofrecieron las dietas a niños con edades de cuatro a cincuenta meses que se encontraran cursando las primeras 48 horas de un episodio de diarrea aguda. Para supervisar la aceptabilidad a la dieta ofrecida, y para poder llevar el registro clínico de las variables de respuesta, se invitó a las madres a mantener a su hijo hospitalizado por la duración del episodio.

Los niños que participaron se seleccionaron a partir de un proceso de vigilancia epidemiológica de morbilidad, que llevaron a cabo auxiliares de campo mediante visitas domiciliarias diarias para identificar a los niños que se encontraran cursando las primeras 24 horas de un episodio diarreico no complicado. La definición inicial de "diarrea" estuvo a cargo de las madres, y se basó en la presencia de evacuaciones de consistencia disminuida en relación con el patrón usual, con un aumento en el número usual de evacuaciones diarias. La presencia de estos signos se corroboró en la clínica, mediante la observación de evacuaciones disminuidas de consistencia y con frecuencia superior a tres en 24 horas. Se invitó a las madres a llevar a sus niños a la clínica para hospitalizarlos a fin de ofrecerles la alimentación en forma supervisada. Al ingresar al hospital cada niño fue objeto de una valoración clínica completa, para confirmar que no había complicaciones agudas de la diarrea que impidieran su participación en el estudio. El peso se registró tanto al ingreso como en forma diaria, hasta que cediera la diarrea o se diera de alta al paciente. La madre fue quien ofrecía los alimentos al niño; si se encontraba ausente, una enfermera se hacía cargo. Se preparó la dieta en forma individual, para cada niño, previo a cada tiempo de comida. Se pesó la cantidad de cada ingrediente ofrecido y se calculó la ingestión mediante la sustracción, a lo ofrecido, de lo que quedaba en el plato después de cada tiempo de alimentación. Asimismo, en cada tiempo de comida se pidió a la madre que juzgara, en forma cualitativa, el apetito que mostraba su niño, aplicando una escala de apreciación de cuatro puntos graduados en una escala tipo Likert, ${ }^{30}$ variando de $0=$ no tiene apetito $\mathrm{a}+++=$ presenta muy buen apetito.

Todos los procedimientos fueron autorizados por el Comité de Investigaciones en Humanos de la institución. Las madres participantes en el estudio hospitalario firmaron consentimiento informado.

\section{A nálisis}

El primer paso en el análisis de los resultados de las entrevistas consistió en una descripción simple de las frecuencias con que cada alimento fue mencionado como preferido o rechazado para ofrecerlo a los niños durante episodios de diarrea aguda. Los resultados del sorteo de alimentos en grupos se organizaron en matrices de co-ocurrencia, que fueron sometidas a un análisis multidimensional. ${ }^{31}$ La matriz de co-ocurrencia se establece mediante la intersección del número de veces que dos alimentos aparecen juntos en un mismo grupo, lo que establece una medida de proximidad entre estos dos alimentos. El análisis multidimensional ofrece la posibilidad de representar en forma gráfica las cercanías o lejanías de los alimentos. A mayor proximidad entre dos alimentos (esto es, entre más veces hayan aparecido juntos en los sorteos), mayor cercanía en su representación gráfica, es más cercana, y viceversa. El análisis multidimensional incluyó dos dimensiones, cuyo sustrato cognitivo se estableció con base en la interpretación que hicieron las mujeres entrevistadas de dichas dimensiones. Finalmente, sobre los resultados del análisis multidimensional se corrió un análisis de componentes principales para identificar qué alimentos estaban más cercanos entre sí, esto es, cuáles pesaban más como componentes de su grupo.

El análisis de los resultados de la fase clínica incluyó la estadística descriptiva (medias, desviación estándar, valores mínimo y máximo) de la ingestión calórica por kilogramo de peso por día, de la ganancia de peso durante la estancia hospitalaria y del tiempo de estancia hospitalaria así como la descripción de los porcentajes con que se apreció el apetito de los niños, por día, de acuerdo con la escala cualitativa empleada. La estadística analítica incluyó el cálculo de la correlación $\left(r^{2}\right)$ entre el tiempo de estancia hospitalaria y la ingestión calórica, entre ésta y la ganancia de peso durante la estancia hospitalaria, y entre la ingestión calórica y el grupo de edad. 


\section{Resultados}

\section{Estudio etnográfico}

Se obtuvieron 142 encuestas, correspondientes a $89 \%$ de las madres seleccionadas para el estudio. El resto de las madres no se encontró en su domicilio en las tres visitas repetidas que hizo el entrevistador. De las madres encuestadas, $75 \%$ dijeron que restringían parcialmente la alimentación a sus niños durante la diarrea, pero que sí ofrecía algunos alimentos durante la enfermedad. El restante $25 \%$ afirmó que mantenía a sus hijos en ayuno completo durante periodos variables cuando se presentaba la fase aguda de la diarrea; dichos periodos variaban de 4 a $108 \mathrm{~h}$ (moda= $24 \mathrm{~h}$, mediana $=36 \mathrm{~h}$ ). Las razones mencionadas, en $61 \%$ de los casos para dicha práctica fueron que "no es bueno que el niño coma cuando tiene diarrea"; $19 \%$ de las madres dijeron que lo hacían "por recomendación médica", y otro porcentaje similar adujo la "pérdida de apetito del niño". En cuanto a las madres que dijeron mantener la alimentación durante la enfermedad, 83\% suspendía sólo algunos alimentos, en tanto que 16\% comentó que mantenía la alimentación usual en forma completa durante la enfermedad.

La frecuencia con que las madres aceptaron o rechazaron el uso de alimentos disponibles localmente para alimentar a los niños durante la diarrea se presenta en el cuadro I. No hubo diferencias estadísticamente significativas entre las comunidades en relación con la aceptabilidad o rechazo de alimentos específicos. Llamó la atención que 33\% de las madres rechazaran ofrecer el seno materno durante el episodio de diarrea aguda. En su mayoría, estas madres fueron jóvenes $(36 \%)$, alfabetas $(70 \%)$, sin predominio de nivel socioeconómico, y adujeron como razones para suspender alimentos durante la enfermedad la creencia de que "no es bueno ofrecer alimentos" (69\%) y el "consejo médico" (24\%).

Los resultados del sorteo de grupos de los alimentos se muestran en el diagrama multidimensional ilustrado en la figura 1; observando la distribución de los alimentos a lo largo del eje de las ordenadas, aquellos que aparecieron en la parte baja del diagrama correspondieron, según la interpretación de las madres, a alimentos que se ofrecen a niños enfermos, y los que aparecen en la parte superior del cuadro correspondieron a la alimentación de niños sanos o en etapa de convalescencia. Observando el esquema en la dimensión horizontal (a lo largo del eje de las abscisas), las madres interpretaron que los alimentos que aparecen a la izquierda eran dados más tempranamente
Cuadro I

Porcentaje de aceptación o rechazo de alimentos OFRECIDOS A NIÑOS MENORES DE CINCO AÑOS DURANTE EPISODIOS DE DIARREA AGUDA POR 142 MADRES ENTREVISTADAS EN OCHO COMUNIDADES RURALES DEL VALle de Solís, MÉxico, 1988-1989

\begin{tabular}{lccc} 
Alimentos* & Aceptado & Rechazado & No contestó \\
Tortilla & 96 & 4 & 0 \\
\hline Carne de pollo & 92 & 8 & 0 \\
\hline Caldo de pollo & 92 & 7 & 1 \\
\hline Caldo de frijol & 82 & 18 & 0 \\
\hline Agua 0 atole de arroz & 81 & 18 & 1 \\
\hline Sopa de pasta & 80 & 20 & 0 \\
\hline Carne de res & 78 & 22 & 0 \\
\hline Arroz cocido & 77 & 21 & 2 \\
\hline Manzana & 77 & 22 & 1 \\
\hline Pan & 76 & 24 & 0 \\
\hline Papa & 74 & 25 & 1 \\
\hline Plátano & 68 & 30 & 2 \\
\hline Leche materna & 66 & 33 & 1 \\
\hline Legumbres & 65 & 34 & 1 \\
\hline Huevo & 54 & 44 & 2 \\
\hline Carne de guajolote & 49 & 49 & 2 \\
\hline Leche de vaca & 37 & 62 & 1 \\
\hline Frijoles & 31 & 69 & 0 \\
\hline N opales & 20 & 80 & 0 \\
\hline Habas & 11 & 88 & 1 \\
\hline Carne de puerco & 4 & 95 & 1 \\
\hline
\end{tabular}

* Disponibles localmente

en el curso de la enfermedad, y los alimentos de la derecha eran ofrecidos más tardíamente. La asociación entre los diversos alimentos se exploró más a fondo mediante el análisis de componentes principales. Las líneas que unen a los diferentes alimentos en la figura 1 muestran la cercanía de los alimentos entre sí, de acuerdo con el análisis de componentes principales. Con base en estos resultados, se diseñaron las dietas que se muestran en el cuadro II.

\section{Estudio clínico}

Se incluyeron 54 niños con edades entre 4 y 50 meses, de los cuales $48 \%$ pertenecían al sexo masculino. El $14.8 \%$ de los niños tenían entre 4 y 6 meses de edad; $40.7 \%$ entre 7 y 12 meses, y $44.4 \%$ era mayor de 12 meses. A su ingreso al hospital todos los niños se encontraban dentro de las primeras 48 horas del episodio diarreico (promedio $19.6 \pm$ desviación estándar de 11.4). En ninguno de ellos se identificó clínicamente 
Caldo de frijol__ Tortilla
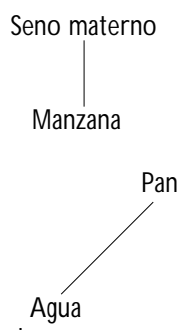

de arroz
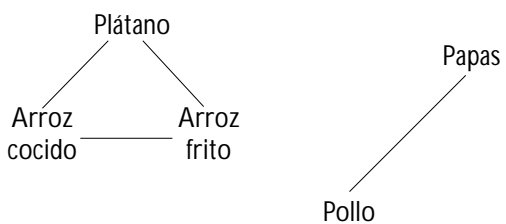

Figura 1. Escala multidimensional QUe muestra las PROXIMIDADES ENTRE ALIMENTOS ESPECÍFICOS, DESTACANDO LA MAYOR CERCANÍA MEDIANTE LÍNEAS QUE UNEN A LOS ALIMENTOS Y QUE RESULTAN DEL ANÁLISIS DE FACTORES SOBRE LOS ALIMENTOS QUE LAS MADRES HAN ACEPTADO ADMINISTRAR A SUS HIJOS DURANTE LOS EPISODIOS DE DIArRea aguda. Valle de Solís, México, 1988-1989

deshidratación, por lo que la alimentación se inició a partir del ingreso. Los niños permanecieron en el hospital un promedio de $47.6 \pm 22.2 \mathrm{~h}$ (mínimo $=17 \mathrm{~h}$, máximo $=104 \mathrm{~h}$ ). En promedio consumieron $44.9 \pm 28.6$ $\mathrm{kcal} / \mathrm{kg} /$ día, mostrando una ganancia promedio de peso de $70.7 \pm 179.7$ g. Sin embargo, $27.8 \%(n=15)$ de los niños mostraron alguna pérdida de peso (con variación entre -20 a $-300 \mathrm{~g}) ; 5.7 \%(\mathrm{n}=3)$ no mostró cambios, y $67.9 \%(\mathrm{n}=36)$ mostró ganancias de peso que fluctuaron de 10 a $500 \mathrm{~g}$. No hubo diferencias estadísticamente significativas entre el grupo de niños que no ganaron peso $(\mathrm{n}=18)$ y los que sí ganaron peso $(n=36)$ en relación con su edad, sexo, puntuación $Z$ de peso/edad, duración en horas de la diarrea al ingreso o número de evacuaciones presentadas desde el inicio del cuadro hasta la admisión. Había un mayor consumo calórico en la medida en que era mayor el grupo de edad $(31.6 \pm 43.9,42.6 \pm 21.2$ y $51.3 \pm 29.9 \mathrm{kcal} / \mathrm{kg} /$ día, para los grupos de 4-6 meses, 7-12 meses y > 12 meses, respectivamente). Hubo una asociación significativa $(p<0.05)$ entre mayor tiempo de estancia en el hospital y mayor ingestión calórica.

La ingestión calórica por kilogramo de peso corporal por día fue mayor conforme aumentó el grupo de edad, con una diferencia estadísticamente significativa $(p<0.05)$ al comparar entre grupos de edad a partir del segundo día de estancia hospitalaria. La ingestión calórica del grupo de menor edad (4-6 meses) fue menor a la encontrada en los otros dos grupos debido a que no se realizó la cuantificación de la ingestión a partir del seno materno.

\section{Cuadro II}

\section{Dietas ofrecidas a los niños durante el episodio de diarRea AGUda.VAlle de Solís, MéxiCo,1988-1989}
ler. día
2do. día
$\geq$ 3er. día

Edad: 4-6 meses: seno materno a libre demanda

\begin{tabular}{llll} 
Mañana & Manzana en puré & Manzana o plátano en puré & Igual + su dieta habitual \\
\hline Tarde & Puré de papa & C aldo de frijol y tortilla & Igual + su dieta \\
\hline N oche & Manzana en puré & Manzana o plátano en puré & Igual + su dieta habitual
\end{tabular}

Edad: 6-12 meses: si aún toma seno materno, continuar a libre demanda; ofrecer agua de arroz a libre demanda (como agua de uso)

\begin{tabular}{llll} 
Mañana & Manzana o plátano rayado o colado & Manzana o plátano rayado y caldo de frijol & Igual + su dieta habitual \\
\hline Colación & Manzana o plátano rayado o colado & Sopa de fideos con tortilla o bolillo & Continuar con los alimentos preferidos \\
\hline Tarde & Caldo de pollo deshebrado con tortilla & $\begin{array}{l}\text { Caldo de pollo y arroz cocido con papa, } \\
\text { plátano, tortilla o bolillo }\end{array}$ & Igual + su dieta habitual \\
& y arroz cocido & Manzana o plátano rayado o colado & Continuar con los alimentos preferidos \\
\hline Colación & Manzana o plátano rayado o colado & Sopa de tortilla & Igual + su dieta habitual
\end{tabular}

Edad: 1-5 años: ofrecer agua de arroz a libre demanda (como agua de uso)

\begin{tabular}{llll} 
Mañana & Manzana o plátano entero, rayado & Manzana o plátano, caldo de frijol y tortilla & Igual + su dieta habitual \\
\hline Colación & Manzana o plátano & Caldo de frijol y tortilla & O frecer alimentos preferidos \\
\hline Tarde & Arroz cocido o guisado & Sopa de pasta, carne de pollo o res, papa, & Igual + su dieta habitual \\
& con pollo, tortilla, plátano & Manzana o plátano & \\
\hline Colación & Caldo de frijol con tortilla & Arroz guisado, plátano, \\
N oche & Caldo de pollo deshehebrado, papa y pan de pollo deshebrado, tortilla & O frecer alimentos preferidos \\
& & & Igual + su dieta habitual
\end{tabular}


La apreciación cualitativa de la ingestión calórica mostró que al ingreso hubo un porcentaje importante $(38.2 \%)$ de niños que mostraban anorexia, la cual desapareció a partir del segundo día de estancia hospitalaria. Asimismo, la proporción de niños que mostraron buen apetito se incrementó marcadamente a partir de las seis horas de estancia hospitalaria, esto es, una vez que se inició la dieta. A partir de las 36 horas de estancia hospitalaria, más de $75 \%$ de los niños mostraba apetito de $++\mathrm{a}+++$ (cuadro IV). Hubo una correlación estadísticamente significativa $(p<0.05)$ entre la apreciación cualitativa del apetito del niño y la ingestión calórica diaria.

\section{Discusión}

El punto de partida de la presente investigación fue el reconocimiento de que la práctica médica en la salud pública debe identificar las características culturales de la población al diseñar intervenciones orientadas a aplicarse en el hogar. ${ }^{32}$ Siguiendo ese concepto, la primera fase del estudio consistió en un estudio exploratorio orientado a conocer las prácticas de las madres en relación con la alimentación de sus hijos durante episodios de diarrea aguda. En primer lugar, destacó que dos terceras partes $(75 \%)$ de las madres no suspendían totalmente la alimentación a sus hijos durante la enfermedad, y que la mayoría (83\%) les ofrecía al menos, algunos alimentos. Este hallazgo fue con- sistente con lo que han notificado otros investigadores de diversos países ${ }^{9,23,33}$ y dio pie para investigar qué alimentos se preferían sobre otros. La exploración etnográfica subsecuente permitió seleccionar alimentos que cumplieran con dos características esenciales para promoverlos como parte de una estrategia de manejo nutricional de la diarrea aguda: la primera fue que estuvieran disponibles localmente, y la segunda que contaran con la aceptabilidad cultural por parte de las madres. De no haber tomado en cuenta esos dos conceptos, probablemente se hubieran promovido alimentos poco aceptados por las madres, comprometiendo desde esta etapa el posible éxito de una intervención educativa. De hecho, al inicio del estudio el grupo de investigación tenía predilección por ciertos alimentos como el nopal, tomando en consideración el reconocimiento de su amplia disponibilidad local, así como de su contenido de carbohidratos y de fibra, que pudieran ayudar, respectivamente, al aporte calórico durante la enfermedad, así como a formar bolo intestinal, que ayudaría a disminuir las evacuaciones líquidas. ${ }^{34} \mathrm{Sin}$ embargo, al interrogar a las madres se encontró que $80 \%$ rechazaba el uso del nopal por asociarlo con una cualidad "fría" del alimento, ${ }^{35,36}$ que resultaba inconveniente para un niño con diarrea.

La selección de los alimentos con aceptabilidad cultural se complementó con el análisis multidimensional del sorteo libre efectuado por las madres. Una de las dos dimensiones identificadas por las madres,

\section{Cuadro III \\ Características clínicas de 54 niños que recibieron alimentación sostenida CON ALIMENTOS DISPONIBLES LOCALMENTE Y ACEPTADOS CULTURALMENTE, DURANTE UN EPISODIO DE DIARREA AGUDA. Valle de Solís, México, 1988-1989}

\begin{tabular}{|c|c|c|c|}
\hline Variable & $\begin{array}{c}4-6 \text { meses } \\
n=8\end{array}$ & $\begin{array}{r}7-12 \text { meses } \\
n=22\end{array}$ & $\begin{array}{c}>12 \text { meses } \\
n=24\end{array}$ \\
\hline \multicolumn{4}{|l|}{ Sexo } \\
\hline Masculino $n(\%)$ & $3(37.5)$ & $12(54.5)$ & $11(45.8)$ \\
\hline Femenino $n(\%)$ & $5(62.5)$ & $10(45.5)$ & $13(54.2)$ \\
\hline Puntaje Z peso/edad ( $x \pm D E)$ & $1.1 \pm .7$ & $-1.6 \pm 1.1$ & $-2.1 \pm 1.0$ \\
\hline Duración en horas de la diarrea al ingreso $(x \pm D E)$ & $23.7 \pm 15.2$ & $19.4 \pm 10.2$ & $18.7 \pm 11.8$ \\
\hline Kcal/kg/día durante la estancia & $31.6 \pm 43.9$ & $42.6 \pm 21.2$ & $51.3 \pm 29.9$ \\
\hline hospitalaria (min., max.)* & $(3,117)$ & $(9,81)$ & $(3,107)$ \\
\hline Ganancia de peso (g) durante & $60.0 \pm 79.8$ & $60.0 \pm 168.0$ & $85.0 \pm 215.4$ \\
\hline la estancia hospitalaria (mín., max.) & $(-20,+200)$ & $(-300,+440)$ & $(-300,+500)$ \\
\hline Tiempo de estancia (h) & $56.5 \pm 29.3$ & $45.4 \pm 24.5$ & $47.4 \pm 17.5$ \\
\hline (mín., max.) & $(28,104)$ & $(21,97)$ & $(17,73)$ \\
\hline
\end{tabular}




\section{Cuadro V}

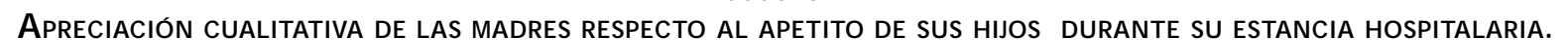
VALLe de Solís, MéXico, 1988-1989

\begin{tabular}{|c|c|c|c|c|c|c|c|c|c|}
\hline Apetito* & $\begin{array}{c}\text { Ingreso } \\
n=34^{\ddagger} \\
(\%)\end{array}$ & $\begin{array}{c}6 \mathrm{~h} \\
\mathrm{n}=47 \\
(\%)\end{array}$ & $\begin{array}{c}12 \mathrm{~h} \\
n=46 \\
(\%)\end{array}$ & $\begin{array}{c}18 \mathrm{~h} \\
\mathrm{n}=40 \\
(\%)\end{array}$ & $\begin{array}{c}24 \mathrm{~h} \\
n=41 \\
(\%)\end{array}$ & $\begin{array}{c}36 \mathrm{~h} \\
n=37 \\
(\%)\end{array}$ & $\begin{array}{c}48 \mathrm{~h} \\
\mathrm{n}=27 \\
(\%)\end{array}$ & $\begin{array}{c}60 \mathrm{~h} \\
\mathrm{n}=16 \\
(\%)\end{array}$ & $\begin{array}{c}72 \mathrm{~h} \\
\mathrm{n}=12 \\
(\%)\end{array}$ \\
\hline \multirow[t]{2}{*}{0} & 13 & 5 & 5 & 2 & 4 & 3 & 2 & 0 & 0 \\
\hline & $(38.2)$ & (10.6) & $(10.9)$ & $(5.0)$ & (9.7) & (8.1) & (7.4) & (0) & (0) \\
\hline \multirow[t]{2}{*}{+} & 4 & 5 & 9 & 5 & 9 & 6 & 3 & 3 & 3 \\
\hline & (11.8) & $(10.6)$ & (19.6) & (12.5) & (21.9) & (16.2) & (11.1) & $(18.8)$ & $(25.0)$ \\
\hline \multirow[t]{2}{*}{++} & 11 & 17 & 15 & 12 & 14 & 11 & 8 & 3 & 3 \\
\hline & $(32.5)$ & $(36.2)$ & $(32.6)$ & $(30.0)$ & $(34.1)$ & (29.7) & $(29.6)$ & (18.8) & $(25.0)$ \\
\hline \multirow[t]{2}{*}{$\overline{t++}$} & 6 & 20 & 17 & 21 & 14 & 17 & 14 & 10 & 6 \\
\hline & $(17.6)$ & $(42.5)$ & $(36.9)$ & $(52.5)$ & $(34.2)$ & (45.9) & (51.8) & $(62.4)$ & $(50.0)$ \\
\hline
\end{tabular}

* Evaluado por las madres mediante una apreciación cualitativa, que fue de 0 = "Sin apetito" a +++= "Muy buen apetito"

₹ El número de niños varía en cada tiempo de evaluación porque no siempre se encontró presente la madre para dar su apreciación sobre el apetito de su hijo

la referida a los alimentos ofrecidos al niño enfermo de acuerdo con su edad, mostró una estrecha semejanza con el proceso de ablactación. En la figura 1 se observa que, partiendo del lado izquierdo al derecho en el diagrama multidimensional, se encuentran el seno materno, la manzana, el agua de arroz, el arroz cocido, el plátano, la sopa de fideos, la tortilla, el pollo, la carne de res y las papas. La otra dimensión, en relación con el proceso de recuperación de la enfermedad, señaló como alimentos preferidos durante la fase aguda a las preparaciones de arroz, frutas (manzana y plátano) y pollo, dejando para la fase de convalescencia, en pautas generales, la sopa de fideos, caldo de frijol, carne de res y tortilla. Esta agrupación reflejó un sentido cultural que no hubiera podido lograrse mediante el diseño de un menú bajo un punto de vista exclusivamente dietético.

La aceptabilidad a esta dieta pudo probarse en el ensayo clínico. Cabe destacar lo temprano que se inició la alimentación controlada de los niños, quienes en promedio se encontraban en el primer día de la enfermedad. Por esta razón, no resultó extraño que el apetito el día del ingreso al hospital se encontrara disminuido, pero aumentó rápidamente con el paso de los días, al tiempo que se mantuvo la alimentación. Varios autores autores han notificado hallazgos similares en relación con la recuperación del apetito. ${ }^{7,37} \mathrm{La}$ ingestión calórica de los niños durante la fase aguda de la enfermedad era inferior a sus requerimientos, ofreciendo apenas alrededor de $45 \%$ de la recomendación dietética de calorías para el grupo mayor de seis meses. ${ }^{38}$ Cabe señalar que en los niños alimentados al seno materno (donde quedaron incluidos todos los menores de seis meses) fue imposible medir el aporte calórico de la leche materna; de ahí la ingestión calórica aparentemente tan baja en esa edad. Sin embargo, la ingestión calórica fue incrementándose conforme pasaron los días, correlacionando con la apreciación cualitativa de las madres sobre el apetito de los niños, y bastó para evitar la pérdida de peso; de hecho, en la mayoría de los casos $(67.9 \%)$ hubo incremento del peso. En otros estudios también se ha encontrado que la alimentación sostenida durante la diarrea promueve la ganancia de peso. Por ejemplo, Brown y colaboradores estudiaron a un grupo de 128 lactantes menores de 36 meses de edad sometidos a diferentes regímenes de alimentación a base de una fórmula láctea elemental durante el proceso diarreico. Tanto los niños que recibieron fórmula completa $(110 \mathrm{kcal} / \mathrm{kg} /$ día $)$ como aquellos a quienes se les administró fórmula a media dilución por dos días, seguida de fórmula completa, a partir del inicio del manejo dietético, mostraron mayor absorción y retención de nitrógeno, reflejada en mayor ganancia de peso, circunferencia braquial y pliegues cutáneos, que los niños que fueron sometidos inicialmente a periodos variables de ayuno. ${ }^{25}$

Los resultados del presente estudio pueden apoyar las intervenciones de manejo dietético del niño con diarrea aguda. Las decisiones de la madre sobre el manejo en el hogar de su hijo enfermo van formando parte de las prácticas culturales de la comunidad, y están influidas por diferentes aspectos, incluyendo el consejo 
de sus mayores (sobre todo de sus madres y sus suegras), su propia experiencia en el manejo de episodios similares anteriores y el consejo médico. ${ }^{6,24,39}$ En este último aspecto es muy importante que el personal de los servicios de salud esté actualizado respecto a las recomendaciones sobre el manejo nutricio del niño con diarrea, de manera que pueda ofrecer una orientación sólida y convincente. Igualmente importante es que la orientación que la madre reciba en los diferentes niveles de atención sea congruente. La recomendación actual de mantener la alimentación durante la enfermedad (siempre y cuando el niño no presente deshidratación u otra complicación de la diarrea que ponga en peligro su vida) $)^{5,26}$ debe apoyarse no sólo en la promoción de los alimentos disponibles localmente y a los cuales el niño se encuentre acostumbrado, sino que debe basarse en una comprensión de la conceptualización cultural de las madres sobre los alimentos ofrecidos a sus niños. Además, las recomendaciones sobre combinaciones específicas de alimentos deben tomar en cuenta tanto las necesidades nutricias del niño como las alteraciones en su fisiología, abarcando tanto la enfermedad como la convalescencia. La pérdida transitoria del apetito durante la fase aguda debe reconocerse para entender el desgano natural del niño enfermo por una dieta completa, lo que puede verse agravado si la dieta que se le ofrece es monótona. De la misma manera, la recuperación del apetito durante la convalescencia debe aprovecharse para favorecer una mayor ingestión de alimentos, tanto en cantidad como en variedad.

El desarrollo de estas recomendaciones debe fundamentarse en investigación formativa enfocada en un entendimiento más amplio de las creencias y prácticas alimentarias de las madres durante la enfermedad y convalescencia de los niños. También debe evaluarse tanto el diseño y aceptación de intervenciones educativas específicas, que busquen ofrecer una dieta más variada y de mayor calidad que la que espontáneamente (esto es, sin intervención) ofrezcan las madres, como el impacto de estas intervenciones sobre la prevención de la desnutrición en los niños. Es deseable que el personal de salud en el campo ofrezca una retroalimentación razonada a los encargados de diseñar las intervenciones, para que éstas se adapten al contexto cultural y promuevan una mejor alimentación. Los resultados de este ejercicio de investigación aplicada podrán servir de modelo para el diseño de otras intervenciones, particularmente de aquellas orientadas a mejorar las prácticas de ablactación.

\section{Referencias}

1. Snyder J, Merson M. The magnitude of the global problem of acute diarrhoeal disease:A review of active surveillance data. Bull W orld $\mathrm{H}$ ealth O rgan 1982;60:605-613.

2. Instituto N acional de Estadística, G eografía e Informática. Anuarios Estadísticos. México, D.F.: IN EGI/SPP, 1990.

3. Dirección General de Epidemiología. Encuesta de Manejo Efectivo de Casos de Diarrea en el Hogar (EMECADI-93). México, D.F.: Secretaría de Salud. Sistema N acional de Encuestas de Salud, 1993.

4. Hirschhorn N . The treatment of acute diarrhea in children. An historical and physiological perspective. Am J C lin N utr 1980;33:637-663.

5. World Health 0 rganization. A manual for the treatment of acute diarrhoea. Ginebra:W HO/CDD/Ser/ 80.2, rev. 1, 1984

6. Martínez $\mathrm{H}$, Tomkins A. N utrition management of diarrhea. Food N utr Bull 1995;16:349-355.

7. Martorell R, Yarbrough C, Yarbrough S, Klein R.The impact of ordinary illnesses on the dietary intakes of malnourished children. Am J C lin N utr 1980;33:345-350.

8. Rahaman M, W ahed M. D irect nutrient loss in diarrhea. En: Chen L, Scrimshaw N S, ed. Diarrhea and Malnutrition. N ueva York: Plenum Press, 1983:157-162.

9. Bentley M, Stallings R, Fukumoto M, Elder J. Maternal feeding behavior and child acceptance of food during diarrhea, convalescence, and health in the central Sierra of Peru. Am J Public Health 1991;81:43-47.

10. Brown K, Pérez F. Determinants of dietary intake during childhood diarrhea and implications for appropriate nutritional therapy. Acta Paediatr 1992;381:127-132.

11. Molla AM, Rahim A, Sarker SA, Mozasfar Z y Rahaman M et al. Intake and absorption of nutrients in children with cholera and rotavirus infection during acute diarrhea and after recovery. N utr Res 1982;2:1233-1242.

12. Sarker S, Molla A, Karim A, Rahaman M. Calorie intake in childhood diarrhea. N utr Rep Int 1982;26:581-590.

13. Molla A, Molla A, Sarker S, Rahaman M. Food intake during and after recovery from diarrhea in children. En: Chen $L$, Scrimshaw N S, ed. Diarrhea and malnutrition. N ueva York: Plenum Press, 1983.

14. Powanda M. Changes in body balances in nitrogen and other key nutrients: D escription and underlying mechanisms. A m J C lin N utr 1977;30: 1254-1268.

15. Dossetor J,W hittle H. Protein-losing enteropathy and malabsorption in acute measles enteritis. Br Med J 1975;2:592-593.

16. Castillo-Duran C, Vial P, U auy R. Trace mineral balance during acute diarrhea in infants. J Pediatr 1988;113:452-457.

17. Golden B, Golden M. Plasma zinc, rate of weight gain, and the energy cost of tissue deposition in children recovering from severe malnutrition on a cow's mil or soya protein based diet. Am J C lin N utr 1981;34: 892-899.

18. Hambidge $K$, Hambidge $C$, Jacobs $M$, Baim J. Low level of zinc in hair, anorexia, poor growth and hypogeusia in children. Pediatr Res 1972;6: 868-874.

19. W alravens $P$, H ambidge K. G rowth of infants fed a zinc supplemented formula. Am J C lin N utr 1976;29:1114-1121.

20. C hung A, Viscorova B. The effect of early oral feeding versus early oral starvation on the course of infantile diarrhea. J Pediatr 1948;33:14-22.

21. Chung A.The effect of oral feeding at different levels on the absorption of foodstuffs in infantile diarrhea.J Pediatr 1948;33:1-13.

22. Alarcón P, Montoya R, Pérez F, Dongo J, Brown K. Clinical trial of locally available mixed diet or lactose-free soy formula for the nutritional 
therapy of acute diarrhea in Peruvian children. En: Kim W Y, ed. International C ongress of N utrition. Seoul, Korea: EwhaW omans U niversity, 1989. 23. Torún B. Local common foods in the dietary management of acute diarrhea: Experience in Guatemala. En: Kim W Y, ed. International Congress of N utrition. Seoul, Korea: Ewha W omans University, 1988.

24. Torún $B, C$ hew F. Recent developments in the nutritional management of diarrhea. Practical approaches towards dietary management of acute diarrhea in developing countries. Trans R Soc Trop Med Hyg 1991;85: 12-17.

25. Brown K, Gastañaduy A, Saavedra J, Lembcke J, Rivas D, Robertson $A D$, et al. Effect of continued oral feeding on clinical and nutritional outcomes of acute diarrhea in children.J Pediatr 1988;112:191-200.

26. World Health 0 rganization. The treatment and prevention of acute diarrhea. Practical guidelines. 2a. edición. Ginebra:W HO, 1989.

27. Allen L, Pelto G, C hávez A. The Collaborative Research and Support Program on Food Intake and Human Function: Mexico project. Storrs, CT: U niversity of C onnecticut, D epartment of N utritional Sciences, 1987.

28. Pelto P, Pelto G. Anthropological research:The structure of inquiry. 2a. edición. Cambridge (N Y): C ambridge University Press, 1978.

29. Bernard HR. Research methods in cultural anthropology. 2a. edición. N ewbury Park, CA: SAGE Publications, 1989.

30. Likert R.A technique for the measurement of attitudes. Arch Psychol 1932:140.

31. Romney A, Shepard R, N erlove S. Multidimensional scaling. A pplications. N ueva York: Seminar Press, 1972.
32. Martínez $\mathrm{H}, \mathrm{H}$ abicht JP. A program to develop culturally and medically sound home-based practices for fluid management of children with acute diarrhea. Food N utr Bull 1996;17:117-119.

33. Huffman S, López RG, Madrid S, Brown K, Bentley M, Black R. Do child feeding practices change due to diarrhoea in the central Peruvian highlands? J Diarrheal D is Res 1991;9:295-300.

34. Hernández $M, C$ hávez $A$, Bourges $H$.Valor nutritivo de los alimentos mexicanos. Publicación L-12, División de Nutrición. México, D.F.: Instituto N acional de la N utrición Salvador Zubirán, 1981.

35. Currier R.The hot-cold syndrome and symbolic balance in Mexican and Spanish-American folk medicine. Ethnology 1966;5:251.

36. Messer E. Hot-cold classification:Theoretical and practical implications of a Mexican study. Soc Sci Med 1981;15B:133-145.

37. Brown K, Stallings R, Creed H, Lopez-de-Romana G, Black R. Effects of common illnesses on infants energy intakes from breast milk and other foods during longitudinal community-based studies in Huascar (Lima), Peru. Am J C lin N utr 1990;52:1005-1013.

38. Subcommittee on the Tenth Edition of the RDA. Recommended dietary allowances. Food and N utrition Board.W ashington, D.C .: N ational A cademy Press, 1989.

39. Ketsela T, A sfaw M, Kebede O. Patterns of breast feeding in western Ethiopia and their relationship to acute diarrhea in infants. JTrop Pediatr 1990;36:180-183. 